\title{
Alterstice
}

Revue internationale de la recherche interculturelle

International Journal of Intercultural Research

Revista International de la Investigacion Intercultural

\section{Violence conjugale et diversité culturelle}

\section{Ghayda Hassan}

Volume 3, numéro 2, 2013

Violence conjugale et diversité culturelle

URI : https://id.erudit.org/iderudit/1077515ar

DOI : https://doi.org/10.7202/1077515ar

Aller au sommaire du numéro

\section{Éditeur(s)}

Alterstice

ISSN

1923-919X (numérique)

Découvrir la revue

Citer ce document

Hassan, G. (2013). Violence conjugale et diversité culturelle. Alterstice, 3(2), 5-7. https://doi.org/10.7202/1077515ar

\section{Résumé de l'article}

La violence conjugale en contexte de diversité culturelle est un phénomène extrêmement complexe à aborder en prévention comme en intervention en raison de ses multiples composantes et qui demeure mal connu et peu documenté. Ce numéro thématique de la revue Alterstice tente de faire un premier pas pour combler ces multiples lacunes, en offrant un espace de réflexion et de partage des connaissances théoriques, cliniques et empiriques autour de la violence conjugale vécue par les femmes immigrantes, réfugiées ou issues des minorités ethnoculturelles. Notre sélection d'articles permet d'amorcer une réflexion complexe et critique sur l'expérience de la violence conjugale, réflexion qui porte sur les différentes sphères de l'intime de la culture familiale, qui touche aussi les cultures et les barrières propres au système de soins et qui atteint enfin les enjeux politiques liés à cette question. Nous espérons que ce numéro thématique constituera une passerelle de sens permettant de bâtir des ponts entre différents univers culturels, pour permettre de développer une relation d'aide fondée sur la complexité des sens et le souci d'assurer une sécurité culturelle, et non une relation de pouvoir, de menace et de contrainte additionnelle pour la femme victime de violence conjugale. 


\section{7}

alterstice

Revue Internationale

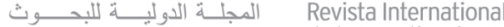

Revista Internacional

International Journal

de la Recherche Interculturelle

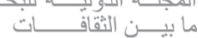

de la Investigacion Intercultural

de Pesquisa Intercultural

of Intercultural Research

INTRODUCTION THÉMATIQUE

\section{Violence conjugale et diversité culturelle}

Ghayda Hassan $^{1}$

\section{Résumé}

La violence conjugale en contexte de diversité culturelle est un phénomène extrêmement complexe à aborder en prévention comme en intervention en raison de ses multiples composantes et qui demeure mal connu et peu documenté. Ce numéro thématique de la revue Alterstice tente de faire un premier pas pour combler ces multiples lacunes, en offrant un espace de réflexion et de partage des connaissances théoriques, cliniques et empiriques autour de la violence conjugale vécue par les femmes immigrantes, réfugiées ou issues des minorités ethnoculturelles. Notre sélection d'articles permet d'amorcer une réflexion complexe et critique sur l'expérience de la violence conjugale, réflexion qui porte sur les différentes sphères de l'intime de la culture familiale, qui touche aussi les cultures et les barrières propres au système de soins et qui atteint enfin les enjeux politiques liés à cette question. Nous espérons que ce numéro thématique constituera une passerelle de sens permettant de bâtir des ponts entre différents univers culturels, pour permettre de développer une relation d'aide fondée sur la complexité des sens et le souci d'assurer une sécurité culturelle, et non une relation de pouvoir, de menace et de contrainte additionnelle pour la femme victime de violence conjugale.

\section{Rattachement des auteures}

${ }^{1}$ Université du Québec à Montréal, Montréal, Canada

\section{Correspondance}

hassan.ghayda@uqam.ca

\section{Mots clés}

violence conjugale; immigration, culture, intervention, prévention, politique

\section{Pour citer cet article :}

Hassan, G. (2013). Violence conjugale et diversité culturelle. Alterstice, 3(2), 5-7. 
La violence conjugale, définie comme une violence physique, émotionnelle, financière ou sexuelle perpétrée par un partenaire conjugal, constitue l'un des problèmes de santé mondiale les plus importants. Même si certains hommes subissent de la violence conjugale, les femmes demeurent plus à risque d'en connaître les formes les plus sévères, ainsi que de souffrir de conséquences aiguës et chroniques. En effet, les statistiques internationales révèlent des taux de violence physique et sexuelle subie par des femmes dans le cadre de relations intimes allant de $3 \%$ à $62 \%$ selon le pays et la zone recensés (Organisation mondiale de la santé, 2012).

Le phénomène persiste et peut même être amplifié dans un contexte de déplacement forcé, de désastre (guerres, désastres naturels, etc.) ou de refuge (camps de réfugiés, demande de statut de réfugié, etc.) ainsi qu'en contexte migratoire, en raison des multiples facteurs de vulnérabilité rattachés à ces différentes réalités. Or la situation des femmes dans ces contextes demeure mal connue et peu documentée. La violence conjugale en contexte de diversité culturelle est en effet un phénomène extrêmement complexe à aborder en prévention comme en intervention, en raison de ses multiples composantes, dont celles liées à l'histoire prémigratoire, au parcours migratoire, aux défis de l'intégration dans la nouvelle société et aux violences structurelles de cette dernière envers les immigrants ou les réfugiés, auxquelles s'ajoutent des barrières d'accès aux soins liées à des caractéristiques culturelles, religieuses, intercommunautaires, sociales et politiques.

Les outils de dépistage de la violence conjugale, tout comme les différentes stratégies d'évaluation, de prévention et d'intervention qui ont été implantées en Amérique du Nord et en Europe, ont été principalement créés et évalués auprès d'une population non spécifique, et ils comportent parfois des stéréotypes et des préjugés essentialisants pour de nombreuses femmes immigrantes, réfugiées ou issues de minorités ethnoculturelles. Leur applicabilité et leur adéquation aux besoins des populations immigrantes demeurent inconnues, tout comme leur efficacité à réduire la violence conjugale et les conséquences qui y sont associées auprès de ces mêmes populations. Par exemple, même lorsqu'une histoire de violence conjugale est dépistée, on ne propose pas systématiquement à ces femmes un suivi psychosocial (Macmillan, Wathen, Jamieson et collab., 2009). On constate ainsi de nombreuses barrières à l'accès aux soins, et le risque de pathologisation de la souffrance et de vulnérabilisation accrue de ces femmes en est potentiellement multiplié.

Les questions demeurent donc nombreuses. Quel est l'état de la situation en ce qui a trait à la violence conjugale en contexte de diversité ethnoculturelle? Quels sont les facteurs de vulnérabilité propres aux femmes en situation de minorité ethnoculturelle? Quelles sont les conséquences de cette violence sur ces femmes et leurs familles? Quels besoins expriment-elles? Comment conçoivent-elles cette violence en lien avec leur passé et leurs conditions de vie actuelles? Comment dépister ou évaluer cette violence? Quelles sont les barrières dans l'accès aux programmes de prévention et aux soins? Les programmes d'intervention offerts répondent-ils aux besoins de ces victimes ou les fragilisent-ils davantage? Ces questions ne constituent qu'un fragment des interrogations liées au manque de connaissances dans le domaine de la violence conjugale auprès des femmes immigrantes, réfugiées et issues des minorités ethnoculturelles.

Ce numéro thématique de la revue Alterstice tente de faire un premier pas pour combler ces multiples lacunes, en offrant un espace de réflexion et de partage des connaissances théoriques, cliniques et empiriques autour de la violence conjugale vécue par les femmes immigrantes, réfugiées ou issues des minorités ethnoculturelles. Notre sélection d'articles permet d'amorcer une réflexion complexe et critique sur l'expérience de la violence conjugale, réflexion qui porte sur les différentes sphères de l'intime de la culture familiale, qui touche aussi les cultures et les barrières propres au système de soins et qui atteint enfin les enjeux politiques liés à cette question.

Nous présentons d'abord quatre articles portant à la fois sur le commun de l'expérience subjective de la violence et sur les réalités spécifiques de femmes provenant d'horizons variés: le Brésil, l'Inde, le Vietnam et le Québec autochtone. Ainsi, dans "Nature of domestic/family violence and barriers to using services among Indian immigrant women ", Colucci, O'Connor, Field, Baroni, Pryor et Minas nous renseignent sur la nature de la violence conjugale et les barrières dans l'accès aux soins pour des immigrantes d'origine indienne en Australie. Leur parole trouve sa source ici à travers une intervention de type théâtre forum, qui permet de lever le tabou associé à la violence conjugale et de rendre compte de cette dernière comme d'une réalité touchant l'ensemble des membres de la communauté et pas simplement la femme qui la subit. Dans "De la plainte aux soins : le parcours des 
victimes de violence conjugale au Brésil », Martins-Borges, Sevegnani Mayorca et Silva Livramento nous font découvrir les nombreuses barrières parsemant le parcours de victimes de violence conjugale au Brésil. Par le biais d'un programme d'intervention psychologique, ces femmes réussissent à se réapproprier certains éléments de leur histoire personnelle, à revaloriser leur identité et à repérer les particularités culturelles leur ayant permis de faire face à ces situations et celles ayant parfois représenté un blocage dans leur processus de changement. Kwiatkowski démontre quant à elle l'importance d'examiner le problème de violence conjugale de manière complexe en tenant compte de la multidimensionnalité de ce phénomène et en évitant toute pensée simpliste. L'auteure le fait en intégrant dans son article "Domestic violence, ethnic diversity, and political and economic change in an upland community of Vietnam » une analyse des aspects politiques, sociaux et économiques dans la compréhension de la violence conjugale au Vietnam. Enfin, Flynn, Lessard, Brassad et Montminy abordent dans « Sortir de la violence, sans sortir de l'autochtonie : l'importance de mieux comprendre les besoins des femmes autochtones en milieu urbain " l'épineuse question de la violence conjugale chez les femmes autochtones en milieu urbain québécois, mettant en lumière les différentes manifestations des rapports de pouvoir en jeu dans la construction des difficultés et des demandes d'aide pour ce groupe de femmes doublement vulnérable.

Une seconde série d'articles touche la question, essentielle, de l'adéquation des services et de leur adaptation aux réalités et aux besoins des femmes immigrantes ou issues des minorités ethnoculturelles victimes de violence conjugale. Dans «Dépister et orienter les personnes d'origine étrangère usant de violence au sein du couple couple: un défi pour les professionnels du champ social », Lorenz et Fluehmann présentent le point de vue de professionnels travaillant au sein d'institutions sociales susceptibles d'orienter les personnes usant de violence conjugale vers les groupes thérapeutiques. Tout en dénonçant le risque de stéréotypie et de discrimination qui peut être associé au pairage entre origine socioculturelle et violence conjugale, les auteures soulignent la nécessité de renforcer les collaborations intersectorielles afin d'optimiser l'accès aux soins et d'améliorer la qualité des interventions.

Le rôle central que peuvent jouer les médias comme miroir et moteur de préjugés sociaux est souligné par Bouchard et Garneau dans leur article « Les enjeux de la survisibilisation/invisibilisation de la violence envers les femmes issues de l'immigration en contexte familial ». Dans cet article, les auteures illustrent l'excès de visibilité des populations immigrantes dans les journaux lorsqu'il est question de violence envers les femmes, mais leur invisibilité sociale lorsqu'il est question de soins. Enfin, les politiques migratoires touchent les femmes et les familles de manière beaucoup plus directe et vulnérabilisante que ne le laisse croire leur aspect macrosystémique. Castro Zavala en fait l'éloquente démonstration dans " Politiques d'immigration : femmes et violence conjugale

dans le contexte québécois ", en analysant la manière dont les politiques d'immigration interagissent avec d'autres formes d'oppression dans la vie des femmes immigrantes, les rendant ainsi plus vulnérables à la violence conjugale.

Les défis à relever dans le domaine de la violence conjugale et de la diversité culturelle sont encore très nombreux et nous espérons que ce numéro thématique et la diversité des idées qui y sont partagées constitueront une passerelle de sens permettant de bâtir des ponts entre différents univers culturels, pour permettre de développer une relation d'aide fondée sur la complexité des sens et sur le souci d'assurer une sécurité culturelle, et non une relation de pouvoir, de menace et de contrainte additionnelle pour la femme victime de violence conjugale.

\section{Références bibliographiques}

Organisation mondiale de la santé. (2012). Comprendre et lutter contre la violence à l'égard des femmes : la violence exercée par un partenaire intime [en ligne]. Organisation mondiale de la santé. Fiche téléchargeable à partir du lien : http://apps.who.int/iris/bitstream/10665/77432/1/WHO_RHR_12.36_fr.pdf?ua=1

Macmillan, H., Wathen, C., Jamieson, E. et collab. (2009). Screening for intimate partner violence in health care settings: a randomized trial. Journal of the American Medical Association, 302, 493-501. 